\title{
(6) OPEN ACCESS \\ Poison prevention practices and medically attended poisoning in young children: multicentre case-control study
}

\author{
Denise Kendrick, ${ }_{1}^{1}$ Gosia Majsak-Newman, ${ }^{2}$ Penny Benford, ${ }^{1}$ Carol Coupland, ${ }^{1}$ \\ Clare Timblin, ${ }^{1}$ Mike Hayes, ${ }^{3}$ Trudy Goodenough, ${ }^{4}$ Adrian Hawkins, ${ }^{5}$ Richard Reading ${ }^{2}$
}

\begin{abstract}
- Additional material is published online only. To view please visit the journal online (http://dx.doi.org/10.1136/ injuryprev-2015-041828).

${ }^{1}$ Division of Primary Care, School of Medicine, University of Nottingham, Nottingham, UK

${ }^{2}$ Norfolk and Norwich University Hospital, Norwich Norfolk and Norwich University Hospitals NHS Foundation Trust, Norwich, UK

${ }^{3}$ Child Accident Prevention Trust, Barnet,UK

${ }^{4}$ Centre for Child \& Adolescent Health, Health and Applied Sciences, University of the West of England, Bristol, UK ${ }^{5}$ Great North Children's Hospital, Newcastle upon Tyne Hospitals NHS Foundation Trust, Newcastle upon Tyne, UK
\end{abstract}

\section{Correspondence to} Professor Denise Kendrick, Division of Primary Care, School of Medicine, University of Nottingham, Tower Building, University Park, Nottingham NG7 2RD, UK; denise. kendrick@nottingham.ac.uk

Received 26 August 2015 Revised 24 March 2016 Accepted 20 April 2016 Published Online First 4 November 2016

\section{ABSTRACT}

Introduction Childhood poisonings are common, placing a substantial burden on health services. Casecontrol studies have found inconsistent evidence about modifiable risk factors for poisonings among children aged $0-4$ years. This study quantifies associations between poison prevention practices and medically attended poisonings in children aged $0-4$ years.

Methods Multicentre case-control study conducted at hospitals, minor injury units and family practices from four study centres in England between 2010 and 2013. Participants comprised 567 children presenting with unintentional poisoning occurring at home and 2320 community control participants matched on age, sex, date of event and study centre. Parents/caregivers provided data on safety practices, safety equipment use, home hazards and potential confounders by means of self-completion questionnaires. Data were analysed using conditional logistic regression.

Results Compared with community controls, parents of poisoned children were significantly more likely not to store medicines out of reach (adjusted OR (AOR) 1.59; 95\% Cl 1.21 to 2.09; population attributable fraction (PAF) 15\%), not to store medicines safely (locked or out of reach (AOR 1.83; 95\% Cl 1.38 to 2.42; PAF 16\%) and not to have put all medicines (AOR 2.11; $95 \% \mathrm{Cl}$ 1.54 to 2.90; PAF 20\%) or household products (AOR $1.79,95 \% \mathrm{Cl} 1.29$ to 2.48 ; PAF $11 \%$ ) away immediately after use.

Conclusions Not storing medicines out of reach or locked away and not putting medicines and household products away immediately after use increased the odds of secondary care attended poisonings in children aged 0-4 years. If associations are causal, implementing these poison prevention practices could each prevent between $11 \%$ and $20 \%$ of poisonings.

\section{INTRODUCTION}

Childhood poisonings are a global problem resulting in considerable health service use. In the USA between 2000 and 2006, unintentional poisonings were the eighth leading cause of non-fatal injuries in children aged $0-4$ years. ${ }^{1}$ In the UK, there are an estimated 26000 emergency department (ED) attendances each year ${ }^{2}$ and there were more than 5200 hospital admissions in 2012-2013 in children aged 0-4 years. ${ }^{3}$ In Australia in 2009-2010, onefifth of all unintentional medicinal poisonings and a tenth of all unintentional poisonings from other substances occurred in children aged 0-4 years. ${ }^{4}$ Childhood poisoning costs the UK National Health
Service (NHS) an estimated $£ 2$ million every year ${ }^{5}$ and in the USA in 2010, total lifetime medical costs for unintentional poisonings in children aged 0-4 years attending ED were estimated at \$66 million. ${ }^{6}$

A small number of case-control studies have explored risk factors for unintentional poisoning in children aged $0-4$ years; several found significant associations with a limited number of poison prevention practices, ${ }^{7-9}$ while others have not. ${ }^{10} 11$ This study therefore aimed to quantify associations between poison prevention practices and medically attended poisonings among children aged $0-4$ years.

\section{METHODS}

The methods are described in the published protocol. $^{12}$ Parental consent to participate was assumed by return of completed study questionnaires.

\section{Study design and setting}

This is one of five case-control studies (the others being falls from furniture, falls on one level, stair falls and burns $)^{13-15}$ conducted simultaneously within EDs, inpatient wards and minor injury units (MIUs) (services external to acute hospitals, treating a limited range of non-serious injuries) in four areas of England: Nottingham, Derby and Lincoln; Bristol; Norwich, Cromer and Great Yarmouth and Newcastle upon Tyne and Gateshead. Cases were recruited between 14 June 2010 and 14 February 2013. Control recruitment commenced at the same time as case recruitment and ended within 4 months of case recruitment.

\section{Participants}

Cases were children aged 0-4 years with poisoning or suspected poisoning (hereafter referred to as poisoning) occurring at home attending an ED or MIU or admitted to hospital. Cases were identified by clinical staff during or following attendance/ admission, using diagnoses recorded in medical records. Eligibility queries were discussed and agreed between researchers at the four study centres. Study questionnaires recorded whether children had swallowed medicinal or household products. Information on suspected poisonings (where it was unclear if a child had ingested a substance) was ascertained from parents' free-text descriptions of poisoning events and from recruitment logs completed by clinical staff. Children with intentional or fatal injuries or living in residential care were excluded. Parents/guardians of potentially eligible cases were approached during their medical
To cite: Kendrick $D$,
Majsak-Newman $G$, Benford $\mathrm{P}$, et al. Inj Prev 2017;23:93-101. 
attendance or by telephone or post within 72 hours of attendance.

Community controls were aged $0-4$ years without a medically attended poisoning on the date of the case's poisoning, recruited from the case's family practice (or neighbouring practice) and not living in residential care. We aimed to recruit an average of four controls per case, individually matched on age (within 4 months of age of case), sex and date of event (within 4 months of case poisoning). Ten controls were identified from the practice register for each case and invited to participate by post. Where more than 10 met inclusion criteria, the 10 with dates of birth closest to that of the case were chosen. Where fewer than four controls were recruited per case, we used controls from cases with more than four controls, controls that were no longer matched to cases (eg, the case had subsequently been excluded) and controls from the other ongoing casecontrol studies as extra controls. These were matched on age (within 4 months of case of age), sex, date of event (within 4 months of case's poisoning) and study centre and were only used once as extra controls. We also used an additional group of cases with other injuries (children admitted to hospital or attending ED) from the other ongoing case-control studies as unmatched hospital controls. Detailed information about inclusion and exclusion of cases and controls who had already been recruited to one of the case-control studies is given in our published protocol. ${ }^{12}$

\section{Outcomes}

The outcome of interest was medically attended poisoning or suspected poisoning from medicines, cleaning agents, garden chemicals and other household products occurring at the child's home resulting in hospital admission, ED or MIU attendance. Poisonings from garden plants were excluded.

\section{Exposures}

Exposures of interest are shown in box 1.

\section{Confounders}

As some controls were not recruited from their matched case family practice, all analyses were adjusted for the Index of Multiple Deprivation (IMD) ${ }^{16}$ (linear term) and distance between postcodes of home and hospital ${ }^{17}$ (quintiles of $\mathrm{km}$ : $\leq 2.1,2.2-3.3,3.4-4.7,4.8-8.8,>8.8$ ). The IMD is a small (400-1200 households) area-based measure of deprivation comprising seven domains (income, employment, health and disability, education skills and training, barriers to housing and services, living environment and crime).

We used directed acyclic graphs (DAGs) to identify the minimal set of confounders for adjusting multivariable models. ${ }^{18-20}$ DAGs included age, sex, IMD and distance from hospital as adjusted variables and potential confounding variables which included number of children in family, ethnic group (white/other), single adult household (yes/no), first child (yes/ no), infant and early Child Behaviour Questionnaire (CBQ) score (activity and high-intensity pleasure subscales) ${ }^{21-23}$ (linear term), Hospital Anxiety and Depression scale (HADS) ${ }^{24}$ (linear term), Parenting Daily Hassles (PDH) scale (parenting tasks subscale $)^{25} 26$ (linear term), hours of out-of-home childcare per week (linear term) and ability to access poisons measured using nine questions, with three-point Likert scale responses from 'not likely' to 'very likely' (grouped as 'all responses not likely', 'at least one quite likely but none very likely', 'at least one very likely'). Exposures were also included in DAGs as confounders for other exposures.

\section{Box 1 Measurements of exposure}

\section{A. Safety equipment use}

1. Safety gates across kitchen doorway*

2. Child resistant closures (CRCs) or blister packs on all medicines*

3. CRCs on all household products*

4. All medicines stored in locked cupboard, medicine cabinet, drawer or fridge*

5. All medicines stored in locked box*

6. All household products stored in locked cupboard or drawer*

B. Hazards

1. Use of baby walkers (ages 0-36 months only)*

2. Presence of things child could climb on to reach high surfaces*

3. Any medicines transferred to different container*

4. Any household products transferred to different container*

C. Safety behaviours

1. All medicines stored out of reach (at adult eye level or above)*

2. All household products stored out of reach (at adult eye level or above)*

3. All medicines stored safely (out of reach or locked or none in house ${ }^{*} \dagger$

4. All household products stored safely (out of reach or locked or none in house) ${ }^{*} \dagger$

5. Returning medicines to usual storage place immediately after use $\ddagger$

6. Returning household products to usual storage place immediately after use‡

7. Teaching rules about what to do/not do when child sees cleaning products $\S$

8. Teaching rules about what to do/not do when medicines are on worktop§

* Exposures measured over the 24 hours prior to case poisoning or 24 hours prior to questionnaire completion for controls with yes/no response options.

tComposite exposure variables for all medicines or all household products stored safely combined responses for storage out of reach, locked and having none in house. Storage was defined as safe if all medicines (or household products) were either locked away or stored out of reach or there were none in the house, and all other combinations were considered unsafe.

‡Exposures measured over the 7 days prior to case poisoning or 7 days prior to questionnaire completion for controls with every time/most times/sometimes/never/not applicable response options. Responses were grouped into 'at least some not put away every time' versus 'all put away every time'. 'Not applicable' responses were excluded from the analysis. $\S$ Exposures had yes/no response options and no time period was specified.

\section{Measurement of exposures and confounders}

Data on exposures, potential confounding variables, sociodemographic, child health and quality of life (PedsQL) ${ }^{27}$ (listed in table 1), injuries and treatment received were ascertained from age-specific parent completed questionnaires (0-12 months, 13-36 months, $\geq 37$ months). Questionnaires measured all exposures across the five ongoing case-control studies. 
Table 1 Characteristics of cases and community controls (percentage, unless stated otherwise)

\begin{tabular}{|c|c|c|}
\hline Characteristics & Cases $\mathrm{n}=567$ & Community controls $n=2320$ \\
\hline \multicolumn{3}{|l|}{ Study centre } \\
\hline Nottingham & $193(34.0)$ & $738(31.8)$ \\
\hline Bristol & 179 (31.6) & $794(34.2)$ \\
\hline Norwich & $106(18.7)$ & $467(20.1)$ \\
\hline Newcastle & $89(15.7)$ & $321(13.8)$ \\
\hline Median age in years (IQR)* & $2.18(1.49,2.92)$ & $2.24(1.54,3.02)$ \\
\hline \multicolumn{3}{|l|}{ Age group: } \\
\hline 0-12 months & $65(11.5)$ & $204(8.8)$ \\
\hline $13-36$ months & $378(66.7)$ & $1575(67.9)$ \\
\hline $37-62$ months & $124(21.9)$ & $541(23.3)$ \\
\hline Male & $280(49.4)$ & $1210(52.2)$ \\
\hline Ethnic group: white & $514(92.1)[9]$ & $2115(92.6)[36]$ \\
\hline Children aged $0-4$ years in family & [11] & {$[29]$} \\
\hline 0 & $6(1.1)$ & $16(0.7)$ \\
\hline 1 & $299(53.7)$ & $1379(60.2)$ \\
\hline 2 & $229(41.2)$ & $810(35.4)$ \\
\hline$\geq 3$ & $22(4.0)$ & $86(3.8)$ \\
\hline First child & 210 (41.7) [64] & $895(42.7)[222]$ \\
\hline Maternal age $\leq 19$ at birth of first child $t$ & $84(16.5)[8]$ & $208(9.7)[14]$ \\
\hline Single adult household & $92(16.6)[13]$ & $262(11.5)[43]$ \\
\hline Median weekly hours out-of-home child care (IQR) & $12(0.5,22.0)[31]$ & $15(2.5,24.0)[112]$ \\
\hline Adults in paid work & [11] & [35] \\
\hline$\geq 2$ & $263(47.3)$ & $1281(56.1)$ \\
\hline 1 & $184(33.1)$ & $742(32.5)$ \\
\hline 0 & 109 (19.6) & $262(11.5)$ \\
\hline Receives state benefits & $228(41.7)[20]$ & $795(35.1)[54]$ \\
\hline Overcrowding (>1 person per room) & $46(8.8)[42]$ & $163(7.4)[128]$ \\
\hline Non-owner occupier & $241(43.5)[13]$ & $771(33.8)[41]$ \\
\hline Household has no car & $81(14.6)[11]$ & $219(9.6)[28]$ \\
\hline Median IMD score (IQR) & $17.5(10.3,31.7)$ & $15.1(9.3,26.5)[24]$ \\
\hline Median distance $(\mathrm{km})$ from hospital (IQR) & $3.5(2.2,5.9)$ & $4.0(2.4,7.6)[24]$ \\
\hline Mean Child Behaviour Questionnaire (CBQ) score (SD) & $4.75(0.91)[24]$ & $4.61(0.86)[186]$ \\
\hline Long-term health condition & $53(9.4)[5]$ & $187(8.1)[21]$ \\
\hline Median Child Health Visual Analogue scale (IQR) & $9.8(8.8,10)[2]$ & $9.6(8.4,10)[14]$ \\
\hline Median health-related quality of life (PedsQL) (IQR)‡ & $\begin{array}{l}n=326[3] \\
91.7(85.7,97.2)\end{array}$ & $\begin{array}{l}\mathrm{n}=1354[24] \\
89.3(82.1,95.2)\end{array}$ \\
\hline Parental assessment of child's ability to access poisons§ & [21] & {$[96]$} \\
\hline All scenarios 'not likely' & $22(4.0)$ & $112(5.0)$ \\
\hline$\geq 1$ scenario 'quite likely' and none 'very likely' & $100(18.3)$ & $513(23.1)$ \\
\hline$\geq 1$ scenario 'very likely' & $424(77.7)$ & $1599(71.9)$ \\
\hline Median Parenting Daily Hassles (PDH) scale (IQR)ף & $14.0(10.3,18.0)[50]$ & $14.0(11.0,18.0)[113]$ \\
\hline Mean Hospital Anxiety and Depression scale (HADS) (SD)ף & $10.9(6.1)[15]$ & $10.8(6.2)[25]$ \\
\hline \multicolumn{3}{|c|}{$\begin{array}{l}\text { [ ] missing values. } \\
\text { *For cases and community controls aged over } 5 \text { years, the case poisoning occurred prior to fifth birthday but study questionnaire was completed after fifth birthday. } \\
\text { †Asked only when mothers completed questionnaire. IMD: higher score reflects greater deprivation. CBQ: higher score reflects more active and intense behaviour. PDH: higher score } \\
\text { reflects more hassle. HADS: higher score reflects greater symptoms of anxiety/depression. Child Health Visual Analogue Scale: range } 0-10 \text {, higher score reflects better health. PedsQL: } \\
\text { measured only in those aged } \geq 2 \text { years, higher score reflects better quality of life. } \\
\text { †Missing values are those with } \geq 50 \% \text { items on any scale missing. } \\
\text { Ability to access poisons is measured by responses to the following questions. How likely do you think it is that your child could: (a) reach or climb onto a worktop; (b) reach or climb } \\
\text { onto something to reach a cupboard at adult eye level; (c) open cupboards, drawers or medicine cabinets with locks or safety catches on them; (d) open a fridge with a lock or safety } \\
\text { catch on it; (e) open a container with a child resistant cap; (f) open a lockable medicine box; (g) get medicines out of blister packs; ( } h \text { ) touch things that you have told him/her not to } \\
\text { and (i) open a safety gate. Response options were very likely, quite likely, not likely, don't know. Responses were categorised as shown in the table. } \\
\text { IMissing values are those with }>1 \text { item missing. } \\
\text { IMD, Index of Multiple Deprivation. }\end{array}$} \\
\hline
\end{tabular}

Home visits by researchers compared observations with selfreports for all exposures across the five studies, which could be ascertained by observation in a sample of 81 cases (including 26 with poisonings) and 81 controls. $^{28}$

\section{Study size}

To detect an OR of 1.59 , with $80 \%$ power, alpha $=0.05$, a correlation between exposures in cases and controls of 0.1 and an average of four controls per case, 266 cases and 1064 controls 
were required, based on exposure prevalence ranging from 21\% (not putting cleaning products away immediately after use) to $65 \%$ (not storing all products safely). The OR of 1.59 was chosen because we considered it the smallest clinically important difference it was feasible to detect within study resources.

\section{Statistical methods}

ORs and 95\% CIs were estimated using logistic regression (conditional for matched analyses), adjusted as described above. Linearity of relationships between continuous confounders and case/control status was ascertained by adding higher order terms to models. We examined if associations varied by child age, gender, ethnicity, housing tenure, unemployment and single parenthood by adding interaction terms to models and assessing significance with likelihood ratio tests $(\mathrm{p}<0.01)$. The population attributable fraction (PAF) per cent was calculated for exposures with statistically significantly raised adjusted ORs (AORs) using a published formula. ${ }^{29}$ As storing medicines safely included storage at adult eye level or above, PAFs for safe storage and storage out of reach are not independent of each other.

For the PedsQL, mean scale scores were computed by summing items and dividing by number of items answered. Means were not computed where $\geq 50 \%$ of items were missing. ${ }^{30}$ We imputed single missing item values for subscales of the HADS using the mean of the remaining six items. Where more than one item was missing, subscale scores were not computed. ${ }^{31}$ We were unable to find missing data guidance for the $\mathrm{PDH}$, so we used the approach used for the HADS. The CBQ allowed missing values and was scored as the total score divided by the number of questions answered. ${ }^{32}$ The main analyses are complete case (CC) analyses, including the single imputed values for the PedsQL, HADS and PDH. We imputed missing data based on all exposure and potential confounding variables (including single imputed values for scales described above) and case/control status to create 20 imputed data sets. These were combined using Rubin's rules. ${ }^{33}$

\section{RESULTS}

Five hundred and sixty-seven cases, 2320 community and 2253 hospital controls participated in the study. The process of recruitment to the study is shown in figure 1 . Response rates were similar for cases $(28.4 \%)$ and community controls (28.3\%). Participants and non-participants were similar by age group and sex $(0-12$ months: $11 \%$ vs $10 \%$; $13-36$ months: $67 \%$ vs $69 \%$; $\geq 37$ months $21 \%$ vs $22 \%$, respectively; $54 \%$ and $53 \%$ male, respectively).

The mean number of community controls per case was 4.09. The median time from date of poisoning to date of

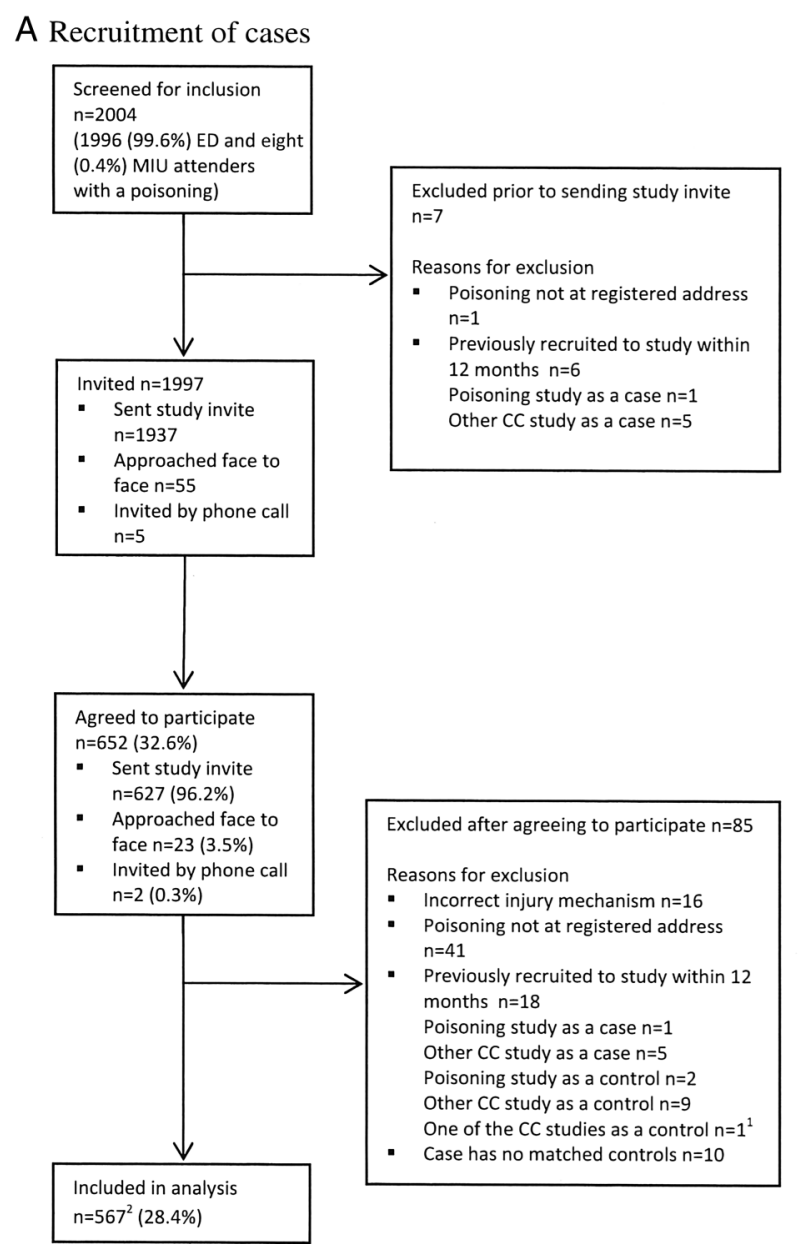

ED, emergency department, MIU, minor injury unit, CC, complete case study ${ }^{1}$ The study the participant had previously been recruited to was not recorded. ${ }^{2}$ Includes eight participants previously recruited to one of the other ongoing $\mathrm{CC}$ studies as a case and 5 previously recruited to this study as a control more than 12 months previously.
B Recruitment of controls

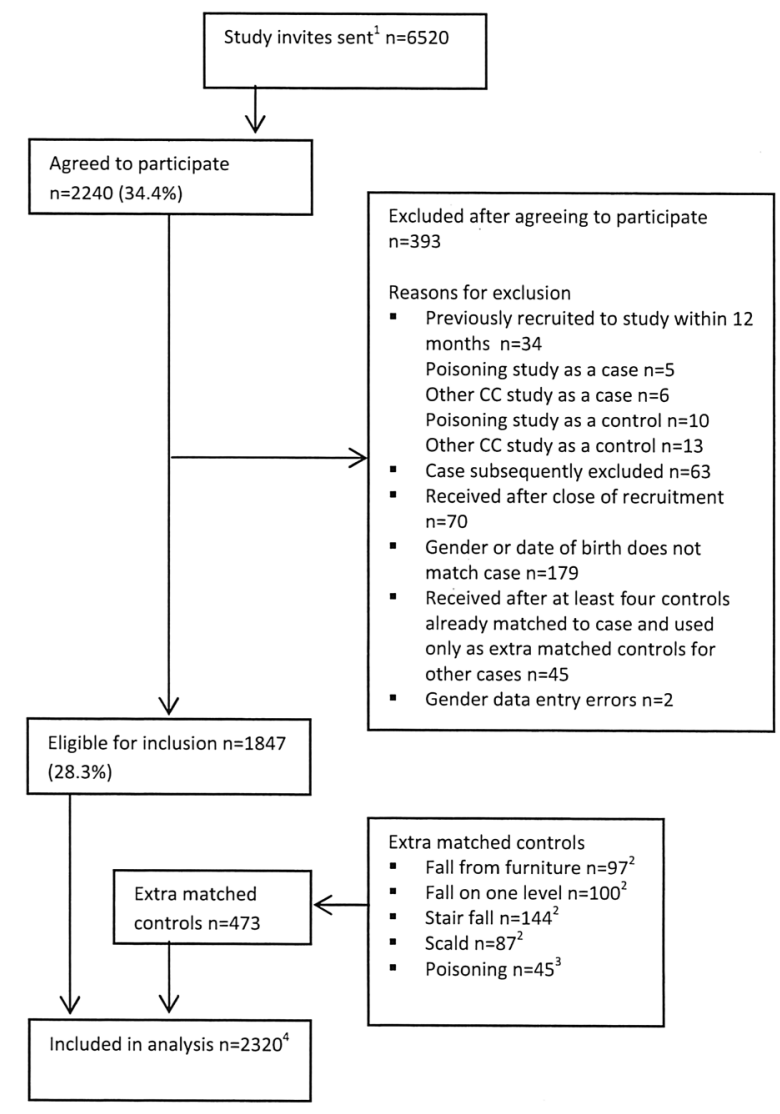

\footnotetext{
${ }^{1}$ Assumed to be $10 x$ number of cases as practices were asked to invite 10 controls for each case.

2 Community controls for cases from the other four ongoing cc studies.

${ }^{3}$ Controls for cases from poisoning study that had more than four controls.

${ }^{4}$ Includes nine participants previously recruited to one of the other ongoing CC studies as a control and one participant previously recruited to this study as a control more than 12 months previously.
} 
Table 2 Age and sex distribution by poisoning agent

Medicinal

poisoning (\%)

Non-medicinal poisoning (\%)

Age in months*
$0-2$

3-5

6-8

9-11

12-23

24-35

36-47

$48-60$

Sex

Male

Female

*One case was aged 60 months. The poisoning occurred prior to the child's fifth

birthday, but study questionnaire was completed after fifth birthday.
$3(1.2)$

$2(0.8)$

$7(2.9)$

$26(10.7)$

$110(45.5)$

$67(27.7)$

$20(8.3)$

$7(2.9)$

$126(52.1)$

$116(47.9)$ questionnaire completion was 12 days (IQR 6, 22). Just over half the cases $57 \%(n=325)$ were medicinal poisonings and $43 \%(n=242)$ were non-medicinal poisonings. Thirty-nine cases $(6.9 \%)$ were suspected poisonings. Table 2 shows poisoning agent by age and sex.

Most cases $(84 \%, n=473)$ were seen and examined but did not require treatment, $6 \%(n=33)$ were treated in $\mathrm{ED}, 8 \%$ $(n=45)$ were admitted to hospital and $2 \%(n=10)$ were discharged from ED and followed up in outpatient clinics or family practices. Cases and community controls were of similar age (2.20 vs 2.24 years). More case than community control families were single adult households $(17 \%$ vs $12 \%)$, with at least one unemployed parent ( $53 \%$ vs $44 \%$ ), receiving state benefits (42\% vs 35\%), living in rented housing (44\% vs 34\%) and in neighbourhoods with higher deprivation scores (17.7 vs 15.1). More mothers of cases than community controls were under the age of 20 when their first child was born $(17 \%$ vs $10 \%)$. More parents of cases than community controls thought their children were very likely to access poisons in at least one of nine scenarios $(78 \%$ vs $72 \%)$. Hospital controls were younger than community controls, had younger mothers, a range of measures indicating greater deprivation, lived closer to hospital and fewer thought their children were very likely to access poisons (see online supplementary table S1).

Table 3 shows that compared with community controls, parents of poisoned children were significantly more likely not to store medicines out of reach (PAF 15\%), were significantly more likely not to store medicines safely (locked or out of reach; PAF 16\%) and were significantly more likely not to have put all medicines (PAF 20\%) and household products (PAF $11 \%$ ) away immediately after use. Compared with community controls, parents of poisoned children were significantly less likely not to have taught their children what to do or not do if medicines were left on the worktop and were significantly less likely not to store all household products safely (locked or out of reach). There were a greater number of significant findings in the analysis using hospital than community controls (see online supplementary tables S2 and S3). These included significantly raised ORs for not having child resistant closures (CRCs) or blister packs on all medicines, not locking all medicines away, not having CRCs on all household products, having things children could climb on to reach high surfaces and not teaching children rules about household products. In addition, ORs for
12 out of 18 exposures for community controls were closer to unity than those for hospital controls.

AORs from CC and multiple imputation (MI) analyses differed by more than $10 \%$ for not storing medicines safely $(11 \%$ lower in MI analysis), not storing all household products safely (18\% higher in MI analysis), transferring cleaning products to different containers $(23 \%$ higher in MI analysis) and not putting household products away immediately after use $(10.1 \%$ lower in MI analysis). Statistical significance differed only for not storing all household products safely (AOR (MI) 0.91, 95\% CI 0.73 to 1.13 ; AOR (CC) $0.77,95 \%$ CI 0.59 to 0.99 ).

There were two significant interactions. First, the odds of poisoning in families not storing household products out of reach were greater in single adult $(2.43,95 \%$ CI 1.09 to 5.43$)$ than two adult households (AOR 0.76, 95\% CI 0.52 to 1.11). Second, the odds of poisoning in families who did not keep medicines locked away were greater in families with male (AOR $1.48,95 \%$ CI 0.85 to 2.58 ) than female cases (AOR $0.59,95 \%$ CI 0.37 to 0.94$)$. These interactions remained statistically significant $(\mathrm{p}<0.01)$ in the MI analyses.

\section{DISCUSSION}

\section{Main findings}

We found not storing medicines out of reach, not storing medicines safely (locked away or out of reach) and not putting medicines and household products away immediately after use increased the odds of secondary care attended poisonings in children aged 0-4 years. If our associations are causal, implementing these poison prevention practices could each prevent between $11 \%$ and $20 \%$ of poisonings.

\section{Strengths and limitations}

To our knowledge, this is the largest case-control study measuring associations between poison prevention practices and medically attended poisoning in young children published until now. We exceeded our sample size requirements, giving the study adequate power to detect ORs smaller than 1.59. The study was conducted in NHS hospitals across England, including urban and rural areas. Analysis included adjustment for a wide range of potential confounders identified using DAGs. ORs for most associations were similar in the CC and MI analyses. Where ORs did differ by more than $10 \%$, the statistical significance of findings differed for only one exposure.

In the validation study, ${ }^{28}$ only three poisoning exposures had at least moderate agreement (medicines kept in fridge $(\mathrm{kappa}=0.54)$, all household products stored at adult eye level or above (kappa=0.48) and having a kitchen safety gate $($ kappa $=0.57))$, and sensitivity and specificity were both $\geq 70 \%$ only for three exposures (all household products stored out of reach, all medicines and household products stored out of reach and use of kitchen safety gates). These relatively low levels of agreement may have resulted from parents reporting 'socially acceptable' responses, or changing prevention practices after their child's injury, after completing the study questionnaire or in anticipation of the home visit. It is also possible that our study questions may not have reliably measured poisoning practices, and further research could help develop better questions. The accuracy of reporting differed significantly between cases and controls for only one exposure (household products transferred to different containers) with controls under-reporting to a greater degree than cases (sensitivity cases $=14 \%$, controls $=0 \%$; specificity cases $=100 \%$, controls $=96 \%$; test for homogeneity $\mathrm{p}=0.03$ ). As non-differential misclassification can result in ORs tending towards unity, ${ }^{34}$ some of our ORs may be 
Table 3 Frequency of exposures in cases and community controls and adjusted odds ratios

\begin{tabular}{llll}
\hline & Cases $\mathrm{n}=567$ & $\begin{array}{l}\text { Community controls } \\
\mathrm{n}=2320\end{array}$ & $\begin{array}{l}\text { Adjusted OR } \\
(95 \% \mathrm{CI}) \S \S\end{array} \quad$ Confounders adjusted fortt \\
\hline
\end{tabular}

\section{Medicines}

All in CRCs/blister packs*

Yes

No

Yes

No

All locked away*

Yes

No

All stored out of reach ${ }^{*} \dagger$

Yes

No

All stored safely ${ }^{*} \ddagger$

Yes

No

Transferred into different container*

No

Yes

All put away immediately after use ${ }^{4}$

Yes

No

Taught child rules about medicines

$$
\text { Yes }
$$

No

Household products

All in CRCs*

$$
\text { Yes }
$$

No

All locked away*

Yes

No

All stored out of reach ${ }^{*} \dagger$

$$
\text { Yes }
$$

No

All stored safely* $¥$

$$
\text { Yes }
$$

No

Transferred into different container*

No

$$
\text { Yes }
$$

All put away immediately after use§

Yes

All in locked box*

$\begin{array}{lcl}459(81.8) & 1991(86.1) & 1.00 \\ 102(18.2)[6] & 321(13.9)[8] & 1.25(0.95,1.65) \\ & & \\ 115(20.5) & 397(17.2) & 1.00 \\ 447(79.5)[5] & 1914(82.8)[9] & 0.82(0.47,1.43)\end{array}$

$89(16.4) \quad 325(14.6) \quad 1.00$

$454(83.6)[24] 0^{* *} \quad 1897(85.4)[92] 6^{* *} \quad 0.91(0.64,1.31)$

$\begin{array}{lll}275(59.3) & 1374(69.2) & 1.00 \\ 189(40.7)[101] 2^{* *} & 612(30.8)[324] 10^{* *} & 1.59(1.21,2.09)\end{array}$

$315(65.6)$

165 (34.4) [87]

1527 (75.1)

1.00

$506(24.9)$ [287]

$1.83(1.38,2.42)$

$533(95)$

$28(5.0)[6]$

$$
\begin{aligned}
& 2206(95.5) \\
& 104(4.5)[10]
\end{aligned}
$$

1.00

$0.96(0.52,1.76)$

$298(58.3)$

$1467(73.8)$

1.00

$213(41.7)[16] 40^{* *}$

$522(26.2)[57] 274^{* *} \quad 2.11(1.54,2.90)$

$304(56.0)$

239 (44.0) [24]

$1108(49.3)$

1138 (50.7) [74]

1.00

$0.66(0.45,0.96)$

$405(72.5)$

$154(27.6)[8]$

$156(30.6)$

$353(69.4)[54] 4^{* *}$

$1620(70.3)$

686 (29.8) [14]

1.00

$0.87(0.69,1.10)$

$614(27.9) \quad 1.00$

$1590(72.1)[106] 10^{* *} \quad 0.90(0.69,1.17)$

81 (16.5)

296 (14)

1.00

$409(83.5)[73] 4^{* *}$

$1823(86.0)[191] 10^{\text {** }}$

\section{$240(50.1)$}

239 (49.9) [88]

948 (45.4)

1138 (54.6) [234]

1.00

$0.77(0.59,0.99)$

\section{$545(97.0)$}

$17(3.0)[5]$

$$
\begin{aligned}
& 2272(98.4) \\
& 38(1.7)[10]
\end{aligned}
$$

1.00

$1.20(0.54,2.65)$

$392(75.0) \quad 1834(82.9) \quad 1.00$

$131(25.0)[30] 14^{* *} \quad 378(17.1)[74] 34^{* *} \quad 1.79(1.29,2.48)$

Did not put all away immediately after use

Taught child rules about

household products

$$
\text { Yes }
$$

$343(63.9)$

194 (36.1) [30]

$1349(60.0)$

$899(40.0)[72]$

1.00

$0.81(0.59,1.12)$

Other exposures

Safety gate to stop child accessing kitchen *
Yes
No

$150(26.7)$

$411(73.3)[6]$
First child, ability to access poisons

CRCs, HADS, PDH, ability to access poisons, hours out-of-home care, first child, medicines locked, medicines put away immediately after use, kitchen safety gate, medicines stored out of reach, things child could climb on to reach high surfaces

CRCs, HADS, PDH, kitchen safety gate, medicines stored out of reach, things child could climb on to reach high surfaces, hours out-of-home care

CRCs, HADS, PDH, ability to access poisons, first child, kitch en safety gate, things child could climb on to reach high surfaces, hours out-of-home care

CRCs, HADS, PDH, ability to access poisons, first child, kitch en safety gate, things child could climb on to reach high surfaces, hours out-of-home care

CRCs, HADS, PDH, locked medicines box, medicines locked, medicines stored out of reach

HADS, PDH, ability to access poisons, first child, medicines locked, medicines stored out of reach, things child could climb on to reach high surfaces

CRCs, HADS, PDH, ability to access poisons, first child, locked medicines box, medicines locked, medicines put away immediately after use, kitchen safety gate, medicines stored out of reach, medicines transferred to different container

First child, ability to access poisons

CRCS, HADS, PDH, kitchen safety gate, products stored out of reach, things child could climb on to reach high surfaces, hours out-of-home care

CRCs, HADS, PDH, ability to access poisons, kitchen safety gate, things child could climb on to reach high surfaces, hours out-of-home care

CRCs, HADS, PDH, ability to access poisons, first child, kitchen safety gate, things child could climb on to reach high surfaces, hours out-of-home care

CRCs, HADS, PDH, products locked, products stored out of reach

HADS, PDH, ability to access poisons, first child, products locked, products stored out of reach, things child could climb on to reach high surfaces

CRCs, HADS, PDH, ability to access poisons, first child, products locked, products stored out of reach, products put away immediately after use, kitchen safety gate, products transferred to different container

HADS, PDH, first child, hours out-of-home care 
Table 3 Continued

\begin{tabular}{|c|c|c|c|c|}
\hline Exposures & Cases $n=567$ & $\begin{array}{l}\text { Community controls } \\
n=2320\end{array}$ & $\begin{array}{l}\text { Adjusted OR } \\
(95 \% \mathrm{CI}) \S \S\end{array}$ & Confounders adjusted fortt \\
\hline \multicolumn{4}{|l|}{$\begin{array}{l}\text { Things child could climb } \\
\text { on to reach high surfaces* }\end{array}$} & CRCs, HADS, PDH, ability to access poisons, first child \\
\hline No & $281(50.0)$ & $1256(54.3)$ & 1.00 & \\
\hline Yes & $281(50.0)[5]$ & $1056(45.7)[8]$ & $1.20(0.93,1.54)$ & \\
\hline $\begin{array}{l}\text { Safety practices measured } \\
\text { only in children aged } \\
0-36 \text { months }\end{array}$ & Cases $n=443$ & Controls $n=1779$ & $\begin{array}{l}\text { Adjusted OR } \\
(95 \% \mathrm{Cl}) \S \S\end{array}$ & Confounders adjusted fort† \\
\hline \multicolumn{4}{|l|}{ Used baby walker* } & HADS, PDH, first child, hours out-of-home care \\
\hline No & $321(75.7)$ & $1225(69.4)$ & 1.00 & \\
\hline Yes & $103(24.3)[19]$ & 539 (30.6) [15] & $0.82(0.61,1.10)$ & \\
\hline \multicolumn{5}{|c|}{$\begin{array}{l}\text { *In the last } 24 \text { hours. } \\
\text { TOut of reach=adult eye level or above. } \\
\text { ¥Safely=out of reach or locked. } \\
\S \text { At least some days in the last week. } \\
\text { IS See definition in footnote to table } 2 \text {. } \\
\text { [missing values] ** not applicable responses } \\
\text { ††All models adjusted for Index of Multiple Deprivation and distance from hospital in addition to listed confounders. } \\
\S \S A n a l y s i s \text { includes single imputed values for hospital anxiety and depression scale and parenting daily hassles scale as described in methods } \\
\text { CRC, child resistant closure; CBQ, Child behaviour questionnaire; HADS, Hospital anxiety and depression scale; PDH=Parenting daily hassles scale. }\end{array}$} \\
\hline
\end{tabular}

underestimated. Our study used community and hospital controls. Hospital controls had a higher prevalence of sociodemographic risk factors and exposures for poisoning than community controls. The greater number of significant findings and the greater magnitude of ORs among hospital controls may therefore reflect Berkson bias.

Participation rates for cases and community controls were low (28\%). Selection bias may have occurred if reasons for nonparticipation were associated with exposures or with secondary care attendance for poisoning. Participation rates were similar by age and sex, but we were not able to measure exposures in non-participants. Most poisonings in our study did not require treatment in the ED, and if seeking medical attention was associated with our exposures, this may have led to ORs being overestimated. Our study included a small number $(n=39)$ of suspected poisonings, which is unlikely to have a major impact on our findings. There may have been residual confounding in our analyses despite adjusting for a range of confounding factors. We did not measure previous poisonings, which could be associated with healthcare behaviour and safety practices, but the number of repeat poisonings is likely to be small. PAFs for storing medicines safely and storing out of reach were not independent of each other and very similar in magnitude; hence, our study suggests storage out of reach may be more important for prevention than locking medicines away.

Our significant interactions should be interpreted with caution due to multiple significance testing and small numbers in subgroups. It is plausible that not locking medicines away increases poisoning risk more among boys than girls, as boys may receive less active supervision than girls. ${ }^{35-37}$ It is also plausible that not storing household products out of reach increases poisoning risk more among single adult households, where supervision may be more challenging or involve more frequent sibling supervision. ${ }^{38} 39$

\section{Comparisons with previous literature}

Three case-control studies report findings consistent with ours. An Australian study of children aged 1-3 years attending an ED following a poisoning and hospital and community controls found accessible storage of medicines in bathrooms increased the odds of poisoning (OR 1.03, 95\% CI 1.002 to 1.080$){ }^{7}$ Two studies using children aged 0-4 years treated in hospital for poisoning and hospital controls found storing toxic substances in boxes/cabinets (OR 3.80, 95\% CI 1.15 to 12.49 ) or $<150 \mathrm{~cm}$ from the floor (OR 16.59, 95\% CI 2.86 to 96.20$)^{8}$, unsafe storage of chemicals and medicines (OR 5.6 95\% CI 1.9 to 16.7) and storing kerosene and petrol in soft drink bottles (OR $3.8,95 \%$ CI 2.0 to 7.3 ) increased the odds of poisoning. ${ }^{9}$ In contrast, two small studies failed to find significant associations between safely packaged household products, ${ }^{10}$ presence of toxic substances, storage practices, frequencies of use, packaging or disposal and poisonings. ${ }^{11}$ Differences in findings between these studies and ours may relate to control groups used, confounders adjusted for, sample size, exposure measurement or types of poisoning.

We found not teaching children safety rules was associated with a lower odds of poisoning. Previous research suggests teaching safety rules can increase the risk of interactions with hazards and injuries in young childre ${ }^{40}$ unless it results in a high level of understanding about the safety issue. ${ }^{41}$ As teaching safety rules is a strategy commonly used by parents, further work should explore relationships between teaching safety rules and poison prevention in young children.

\section{Implications for policy and practice}

If our associations are causal, improving prevention practices, particularly storing out of reach and putting poisons away immediately after use could reduce medically attended poisonings in children aged $0-4$ years. Poison prevention education can be provided during well-child contacts, when prescribing for families with young children and after poisoning events.

\section{What is already known on the subject?}

- Poisonings are common in children aged 0-4 years, placing a substantial burden on health services.

- Previous case-control studies provide inconsistent evidence about modifiable risk factors for unintentional poisonings in children aged $0-4$ years. 


\section{What this study adds?}

- Not storing medicines out of reach or locked away and not putting medicines and household products away immediately after use increased the odds of secondary care attended poisonings in children aged $0-4$ years.

- If associations are causal, implementing these poison prevention practices could each prevent between $11 \%$ and $20 \%$ of poisonings.

Advice should cover cupboard/cabinet lock use and provision of free or low-cost locks for low-income families. Commissioners should ensure child health services include these activities. Increasing effectiveness and durability of cupboard/drawer/ cabinet latches and locks and changes to CRCs, their testing protocols and specifications of products required to be in such containers could also help prevent poisonings.

Correction notice This article has been corrected since it was published Online First. In Table 3, the values in the 'Cases' and 'Community controls' columns have been updated for rows 'Medicines' - 'All put away immediately after use ${ }^{4 \prime}$ and 'Household products' - 'All put away immediately after use ${ }^{4}$.

Acknowledgements We wish to thank the parents who participated in the study We would also like to thank the principal investigators, liaison health visitors, research nurses and other staff from the EDs and MIUs who assisted with recruiting participants from Nottingham University Hospitals NHS Trust, Derby Hospitals NHS Foundation Trust, United Lincolnshire Hospitals NHS Trust, Norfolk and Norwich University Hospitals NHS Foundation Trust, James Paget University Hospitals NHS Foundation Trust, University Hospitals Bristol NHS Foundation Trust, North Bristol Healthcare Trust, Newcastle Upon Tyne Hospitals NHS Foundation Trust, Gateshead NHS Foundation Trust and Northumbria Healthcare NHS Foundation Trust. We gratefully acknowledge the support provided for recruitment by the Primary Care Research Networks for East Midlands and South Yorkshire, Leicestershire, Northamptonshire and Rutland, East of England, Northern and Yorkshire and from South West and Trent, Norfolk \& Suffolk, Northumberland Tyne and Wear and Western Comprehensive Local Research Networks. Written permission has been obtained and we thank Joanne Ablewhite, Philip Miller, Jane Stewart, Persephone Wynn and Ben Young from the University of Nottingham; Lisa McDaid, Clare Ferns and Nathalie Horncastle from the Norfolk and Norwich University Hospitals NHS Foundation Trust; Toity Deave, Pilar Munoz and Benita Laird-Hopkins from the University of the West of England; Paul Hindmarch, Emma Davison and Laura Simms from the Great North Children's Hospital, Newcastle upon Tyne and Bryony Kay from the Bristol Royal Hospital for Children who helped with recruitment, data collection, prepared data for analysis or commented on drafts of papers. We would like to acknowledge the following principal investigators who contributed to obtaining funding, study design, project management in their centres and to interpretation of analyses and commented on drafts of papers: Elizabeth Towner (University of the West of England), Elaine McColl (Newcastle University), Alex J Sutton and Nicola Cooper (University of Leicester) and Frank Coffey (Nottingham University Hospitals NHS Trust). We are also very grateful to Rose Clacy, lay research adviser, who attended project management meetings, helped draft and pilot study documentation, advised on recruitment strategies and commented on drafts of the paper.

Contributors DK, CC, MH and RR designed the study and GM-N, PB, CT, TG and $A H$ contributed to study design. DK and RR supervised the study. GM-N, PB, $\mathrm{CT}$, TG and AH collected data. DK, GM-N, PB, CC and CT analysed data and all authors interpreted data. DK, GM-N, PB and CT drafted sections of the manuscript. All authors critically reviewed the manuscript and approved the final manuscript.

Funding This paper presents independent research funded by the National Institute for Health Research (NIHR) under its Programme Grants for Applied Research Programme (RP-PG-0407-10231). The views expressed in this article are those of the authors and not necessarily those of the NHS, the NIHR or the Department of Health.

Competing interests None declared.

Ethics approval Nottinghamshire research ethics committee 1 (09/H0407/14).

Provenance and peer review Not commissioned; externally peer reviewed.
Open Access This is an Open Access article distributed in accordance with the terms of the Creative Commons Attribution (CC BY 4.0) license, which permits others to distribute, remix, adapt and build upon this work, for commercial use, provided the original work is properly cited. See: http://creativecommons.org/licenses/ by/4.0/

\section{REFERENCES}

1 Borse NN, Gilchrist J, Dellinger AM, et al. CDC childhood injury report: patterns of unintentional injuries among 0-19 year olds in the United States, 2000-2006. Atlanta, GA: Centers for Disease Control and Prevention National Center for Injury Prevention and Control, 2008

2 Department for Trade and Industry. 24th (Final) report of the home and leisure accident surveillance system. 2000, 2001 and 2002 data. London: Department for Trade and Industry, 2003.

3 Health and Social Care Information Centre. Hospital Episode Statistics, Admitted Patient Care, England 2012-13: external causes. http://www.hscic.gov.uk/ catalogue/PUB12566/hosp-epis-stat-admi-ext-caus-2012-13-tab.xlsx (accessed 27 Feb 2015).

4 Tovell A, McKenna K, Bradley C, et al. Hospital separations due to injury and poisoning 2009-10. Injury research and statistics series No. 69. Australia: Australian Government, 2012

5 Child Accident Prevention Trust. Poisoning — how to reduce hospital admissions. http://www.makingthelink.net/news/14-01-13/ poisoning-how-reduce-hospital-admissions (accessed 27 Feb 2015).

6 Centers for Disease Control and Prevention. Data \& Statistics (WISQARSTM): cost of Injury Reports. Nonfatal Emergency Department Treated and Released Injuries, Both Sexes, Ages 0 to 4, United States, 2010. Intent: Unintentional. Mechanism: Poisoning. http://wisqars.cdc.gov:8080/costT/ (accessed 27 Feb 2015).

7 Schmertmann $M$, Williamson $A$, Black $D$, et al. Risk factors for unintentional poisoning in children aged 1-3 years in NSW Australia: a case-control study. BMC Pediatr 2013:13:88.

8 Ramos CL, Barros HMT, Stein AT, et al. Risk factors contributing to childhood poisoning. J Pediatr (Rio J) 2010;86:435-40.

9 Ahmed B, Fatmi Z, Siddiqui AR, et al. Predictors of unintentional poisoning among children under 5 years of age in Karachi: a matched case-control study. Inj Prev 2011; 17:27-32

10 Petridou E, Kouri N, Polychronopoulou A, et al. Risk factors for childhood poisoning: a case-control study in Greece. Inj Prev 1996;2:208-11.

11 Chatsantiprapa K, Chokkanapitak J, Pinpradit N. Host and environment factors for exposure to poisons: a case-control study of preschool children in Thailand. Inj Prev 2001;7:214-17

12 Majsak-Newman G, Benford P, Ablewhite J, et al. Keeping children safe at home: protocol for a matched case-control study of modifiable risk factors for poisoning. Inj Prev 2014;20:e10.

13 Kendrick D, Maula A, Stewart J, et al. Keeping children safe at home: protocol fo three matched case-control studies of modifiable risk factors for falls. Inj Prev 2012;18:e3

14 Kendrick D, Maula A, Reading R, et al. Risk and protective factors for falls from furniture in young children: multicenter case-control study. JAMA Pediatr 2015;169:145-53.

15 Wynn P, Stewart J, Kumar A, et al. Keeping children safe at home: protocol for a case-control study of modifiable risk factors for scalds. Inj Prev 2014;20:e11.

16 Department for Communities and Local Government. English Indices of Deprivation, 2010. https://www.gov.uk/government/statistics/english-indices-of-deprivation-2010 (accessed 29 Oct 2014)

17 Department for Education. Education and skills in your area. Postcode distances. http://www.education.gov.uk/cgi-bin/inyourarea/distance.pl (accessed 29 Oct 2014).

18 Greenland S, Brumback B. An overview of relations among causal modelling methods. Int J Epidemiol 2002;31:1030-7.

19 Greenland S, Pearl J, Robins JM. Causal diagrams for epidemiologic research. Epidemiology 1999;10:37-48.

20 Shrier I, Platt R. Reducing bias through directed acyclic graphs. BMC Me Res Methodol 2008:8:1-15.

21 Gartstein MA, Rothbart MK. Studying infant temperament via the revised infant behavior questionnaire. Infant Behav Dev 2003;26:64-86.

22 Putnam SP, Gartstein MA, Rothbart MK. Measurement of fine-grained aspects of toddler temperament: the early childhood behavior questionnaire. Infant Behav Dev 2006:29:386-401

23 Putnam SP, Rothbart MK. Development of short and very short forms of the children's behavior questionnaire. J Pers Assess 2006:87:102-12.

24 Bjelland I, Dahl AA, Haug TT, et al. The validity of the hospital anxiety and depression scale: an updated literature review. J Psychosom Res 2002;52:69-77.

25 Crnic KA, Booth CL. Mothers' and fathers' perceptions of daily hassles of parenting across early childhood. J Marriage Fam 1991;53:1043-50.

26 Crnic KA, Greenberg MT. Minor parenting stresses with young children. Child Dev/ 1990;61:1628-37. 
27 Varni JW, Seid M, Kurtin PS. PedsQL(TM) 4.0: reliability and validity of the pediatric quality of life inventory version 4.0 generic core scales in healthy and patient populations. Med Care 2001;39:800-12.

28 Watson $\mathrm{M}$, Benford $\mathrm{P}$, Coupland $\mathrm{C}$, et al. Validation of a home safety questionnaire used in a series of case control studies. Inj Prev 2014;20:336-42.

29 Rockhill B, Newman B, Weinberg C. Use and misuse of population attributable fractions. Am J Public Health 1998;88:15-19.

30 Varni JW. The PedsQL Scoring Algorithm. http://www.pedsql.org/score.html (accessed 27 Feb 2015)

31 GL Assessment. The Hospital Anxiety and Depression Scale. Frequently asked questions. How should missing data be treated. http://www.gl-assessment.co.uk/ products/hospital-anxiety-and-depression-scale/hospital-anxiety-and-depressionscale-faqs (accessed 27 Feb 2015).

32 Rothbart M. Mary Rothbart's Temperament Questionnaires. Frequently Asked Questions. http://www.bowdoin.edu/ sputnam/rothbart-temperamentquestionnaires/faq/ (accessed 27 Feb 2015).

33 Rubin DB. Multiple imputation for nonresponse in surveys. New York: John Wiley \& Sons, 1987.

34 Jurek AM, Greenland S, Maldonado G, et al. Proper interpretation of non-differential misclassification effects: expectations vs observations. Int J Epidemio/ 2005:34:680-7.
35 Phelan KJ, Morrongiello BA, Khoury JC, et al. Maternal supervision of children during their first 3 years of life: the influence of maternal depression and child gender. J Pediatr Psychol 2014;39:349-57.

36 Morrongiello BA, Corbett M, McCourt M, et al. Understanding unintentional injury-risk in young children I. The nature and scope of caregiver supervision of children at home. J Pediatr Psychol 2006:31:529-39.

37 Morrongiello BA, Dawber T. Toddlers' and mothers' behaviors in an injury-risk situation: implications for sex differences in childhood injuries. J Appl Dev Psychol 1998; 19:625-39.

38 Schwebel DC, Brezausek CM, Ramey SL, et al. Interactions between child behavior patterns and parenting: implications for children's unintentional injury risk. J Pediatr Psychol 2004;29:93-104.

39 Morrongiello BA, Schell SL, Keleher B. Advancing our understanding of sibling supervision and injury risk for young children. Soc Sci Med 2013;96:208-13.

40 Morrongiello BA., Ondejko L, Littlejohn A. Understanding toddlers' in-home injuries: I. Context, correlates, and determinants. J Pediatr Psychol 2004;29:415-31

41 Morrongiello BA, Widdifield R, Munroe K, et al. Parents teaching young children home safety rules: implications for childhood injury risk. J App/ Dev Psychol 2014;35:254-61.

\section{Why elderly people fall}

A report in Neurology suggests that subtle changes in older people's brain activity may increase the risk of falls by as much as $32 \%$. A total of 166 seniors with no disabilities, dementia or gait problems were attached to a machine that monitored activity of the prefrontal cortex. They were then asked to do tasks such as walking and reciting alternate letters of the alphabet at the same time. Those whose brains worked especially hard during that particular task, possibly to compensate for mild cognitive decline, were much more likely to suffer a fall over the next 4 years.

\section{Bronx building fatal scalding}

The owner of a building in the Bronx, New York, USA where two young girls were scalded to death by a steam blast from a malfunctioning radiator, was named the city's fourth worst landlord last year. The Department of Housing Preservation and Development listed 1263 violations in eight of his buildings. City records show more than US\$10 000 fines over the last decade. The building where the deaths occurred had 24 unresolved violations.

\section{Nez Rouge versus drunk driving}

Nez Rouge is French for red nose, referring not to reindeer, but to the noses of alcohol drinkers. For over 20 years, Canadian Automobile Association (CAA)-Quebec has used volunteer drivers during the Xmas season to transport the intoxicated and thus avoid crashes and penalties. Last year, in Montreal alone, Nez Rouge gave 11408 safe rides home. Across Quebec, the total was 60741 rides involving 10000 volunteers in 61 communities. 